\title{
Holographic heat engine within the framework of massive gravity
}

\author{
Jie-Xiong $\mathrm{Mo}^{1}$ and Gu-Qiang $\mathrm{Li}$ \\ Institute of Theoretical Physics, Lingnan Normal University, \\ Cun Jin Road No. 29, Zhanjiang, China \\ Department of Physics, Lingnan Normal University, \\ Cun Jin Road No. 29, Zhanjiang, China \\ E-mail: mojiexiong@gmail.com, zsgqli@hotmail.com
}

ABSTRACT: Heat engine models are constructed within the framework of massive gravity in this paper. For the four-dimensional charged black holes in massive gravity, it is shown that the existence of graviton mass improves the heat engine efficiency significantly. The situation is more complicated for the five-dimensional neutral black holes since the constant which corresponds to the third massive potential also contributes to the efficiency. It is also shown that the existence of graviton mass can improve the heat engine efficiency. Moreover, we probe how the massive gravity influences the behavior of the heat engine efficiency approaching the Carnot efficiency.

Keywords: Black Holes, Classical Theories of Gravity

ArXiv EPrint: 1707.01235

\footnotetext{
${ }^{1}$ Corresponding author.
} 


\section{Contents}

1 Introduction 1

2 A brief review on thermodynamics of black holes in massive gravity 2

3 Four-dimensional charged black holes in massive gravity 5

4 Five-dimensional neutral black holes in massive gravity 4

$\begin{array}{lll}5 & \text { Conclusions } & 10\end{array}$

\section{Introduction}

The black hole thermodynamics has gained renewed attention from the perspective of the extended phase space [1] where the cosmological constant is identified as thermodynamic pressure. Close relation between AdS black holes and van der Waals liquid-gas system has been further enhanced [2]. And the mass of the black hole gains new physical interpretation as the enthalpy [3] rather than as the internal energy. For a recent review on the extended phase space thermodynamics, one can refer to ref. [4].

Within the framework of the extended phase space, the creative proposal of holographic heat engine was put forward for the first time in ref. [5], allowing the mechanical work to be extracted from heat energy. This proposal has received considerable attention and the effects of Gauss-Bonnet gravity [6], Born-Infeld electrodynamics [7], spacetime dimensionality [8-10] on the heat engine efficiency as well as other interesting aspects [11-19] have been probed. Several months ago, ref. [20] proposed the idea of approaching the Carnot limit at finite power via the heat engine model constructed in the Reissner-Nordström (RN)-AdS black holes spacetime. An ingenious heat engine cycle was considered with the critical point placed at one of the corners. It was shown that both the heat engine efficiency $\eta$ and Carnot efficiency $\eta_{C}$ converge at large $q$. It was further argued that even if the critical point is not on the cycle, the approach $\eta \rightarrow \eta_{C}$ at large $q$ can be achieved.

In this paper, we would like to construct the heat engine within the framework of massive gravity. As we know, gravitons are introduced as massless particles in Einstein gravity. However, there exist several arguments that gravitons should be massive. By including mass terms, massive gravity has served as a straightforward modification of general relativity. Fierz and Pauli first constructed the linear theory of massive gravity [21]. It was reported that Boulware-Deser ghost instability $[22,23]$ exists at the nonlinear level. To overcome this problem, Rham, Gabadadze and Tolley proposed a class of nonlinear massive gravity [24, 25], which was shown to be ghost-free by Hassan [26, 27]. Within this framework, four-dimensional Ricci flat black hole solutions with a negative cosmological 
constant were found [28]. These solutions were generalized to $(n+2)$ charged black holes with any horizon topology [29], with thermodynamics and other related aspects disclosed in refs. [29-32]. For the most recent literatures concerning the black holes in massive gravity, see refs. [33-42], where varieties of peculiar properties were discovered.

The motivation of our research is as follows. Firstly, it is of physical significance in the context of black holes to study heat engines. On the one hand, extracting energy from black holes has long been a challenging and exciting topic ever since the proposal of Penrose process. And the heat engine provides yet another novel mechanism to extract energy via black holes [5]. It was even suspected that they may serve as possible energy source for the high energy astrophysical phenomena near the black hole [16]. On the other hand, the study of heat engine can also act as criterion to discriminate different thermodynamic approaches concerning black holes [9]. Secondly, the research is also interesting in the context of holography. The heat engine cycle was proposed to be interpreted holographically as a journal through a family of large $N$ field theories because changing $\Lambda$ involves changing $N$ in the dual field theory [5]. Choosing black holes in massive gravity as research subject has its own right in holography. It was discovered in the holographic conductor model that the graviton mass can play the same role as the lattice does [28]. Last but not the least, the research in this paper is of interest in the context of massive gravity itself. One may wonder how the graviton mass affects the heat engine efficiency. Since many peculiar properties of black holes have been disclosed in massive gravity, it is natural to expect that generalization of the current research of heat engine to massive gravity may give rise to new findings. It is not merely an exercise that follows closely earlier studies but a promising attempt to pave way for improving the heat engine efficiency.

The organization of this paper is as follows. In section 2 we will review the thermodynamics of black holes in massive gravity. The heat engine model via the four-dimensional black holes in massive gravity will be constructed in section 3 while the five-dimensional black hole case will be discussed in section 4 . In the end, we will present a brief conclusion in section 5 .

\section{A brief review on thermodynamics of black holes in massive gravity}

The action of $(n+2)$-dimensional massive gravity reads [28] (note that the non-trivial part related to massive gravity was proposed in $[24,25])$

$$
S=\frac{1}{16 \pi} \int d^{n+2} x \sqrt{-g}\left[R+\frac{n(n+1)}{l^{2}}-\frac{1}{4} F^{2}+m^{2} \sum_{i=1}^{4} c_{i} \mathcal{U}_{i}(g, f)\right],
$$

where $R, F=F_{\mu \nu} F^{\mu \nu}, m, f$ denote the Ricci scalar curvature, the Maxwell invariant, the massive parameter and the reference metric respectively. And the electromagnetic field tensor $F_{\mu \nu}$ reads $\partial_{\mu} A_{\nu}-\partial_{\nu} A_{\mu}$. In addition to the metric $g_{\mu \nu}$, theories of massive gravity inevitably include another rank-2 symmetric tensor $f_{\mu \nu}$, henceforth called the reference metric. Because one can not construct a mass term via the interaction terms that is formed from the metric alone [27]. 
The main differences between the action of massive gravity and that of Einstein gravity are reflected in the last four massive potential terms associated with the graviton mass. $\mathcal{U}_{i}$ denotes the $i$ th massive potential while $c_{i}$ are the constants corresponding to them. $\mathcal{U}_{i}$ can be fixed by the eigenvalue of the $(n+2) \times(n+2)$ matrix $\mathcal{K}^{\mu}{ }_{\nu} \equiv \sqrt{g^{\mu \alpha} f_{\alpha \nu}}$, where $f_{\mu \nu}$ denotes the reference metric and the square root in $\mathcal{K}$ can be interpreted as $(\sqrt{A})^{\mu}{ }_{\nu}(\sqrt{A})^{\nu}{ }_{\lambda}=A^{\mu}{ }_{\nu}$. The expressions of $\mathcal{U}_{i}$ read $[24,29]$

$$
\begin{aligned}
& \mathcal{U}_{1}=[\mathcal{K}] \\
& \mathcal{U}_{2}=[\mathcal{K}]^{2}-\left[\mathcal{K}^{2}\right] \\
& \mathcal{U}_{3}=[\mathcal{K}]^{3}-3[\mathcal{K}]\left[\mathcal{K}^{2}\right]+2\left[\mathcal{K}^{3}\right] \\
& \mathcal{U}_{4}=[\mathcal{K}]^{4}-6\left[\mathcal{K}^{2}\right][\mathcal{K}]^{2}+8\left[\mathcal{K}^{3}\right][\mathcal{K}]+3\left[\mathcal{K}^{2}\right]^{2}-6\left[\mathcal{K}^{4}\right]
\end{aligned}
$$

where $[\mathcal{K}]=\mathcal{K}^{\mu}{ }_{\mu}$.

It is worth noting that the potential for massive gravity chosen here leads to a theory free of pathologies. On the one hand, this class of nonlinear massive gravity was shown to be ghost-free by Hassan [26, 27]. On the other hand, tachyonic instabilities can also be avoided by choosing the coefficients of the potential properly. One can expand the action to quadratic order around the proposed background to examine whether tachyonic instabilities exist. It was mentioned in the footnote of ref. [29] "the coefficients $c_{i}$ might be required to be negative if $m^{2}>0$ for a self-consistent massive gravity theory. For the AdS space, these restrictions might be a little looser. If the mass square obeys Breitenlohner-Freedman bounds, the fluctuations in AdS space of some fields with negative mass square can still be stable". In this paper, we restrict the choices of coefficients to $m^{2}>0, c_{i}<0(i=1,2,3)$ for nonzero $\mathcal{U}_{i}$ to ensure the absence of tachyonic instabilities.

The static black hole solution of the above action was found with the spacetime metric and reference metric read [29]

$$
\begin{aligned}
& d s^{2}=-f(r) d t^{2}+f^{-1}(r) d r^{2}+r^{2} h_{i j} d x^{i} d x^{j}, \\
& f_{\mu \nu}=\operatorname{diag}\left(0,0, c_{0}^{2} h_{i j}\right),
\end{aligned}
$$

where $c_{0}$ is a positive constant while $h_{i j} d x^{i} d x^{j}$ denotes the line element of an Einstein space with constant curvature $n(n-1) k$. $k$ can be taken as 1,0 , or -1 respectively without loss of generality, which corresponds to spherical, Ricci flat, and hyperbolic topology. Note that this reference metric was generalized from the one proposed in ref. [28], where the spatial reference metric $\operatorname{diag}(0,0,1,1)$ was considered for the purpose that the spatial graviton mass term can preserve general covariance in the $t$ and $r$ coordinates but break it in the two spatial dimensions when a coordinate transformation preserving the $x$ and $y$ coordinates is performed.

$\mathcal{U}_{i}$ and $f(r)$ have been obtained as [29]

$$
\begin{aligned}
& \mathcal{U}_{1}=n c_{0} / r, \\
& \mathcal{U}_{2}=n(n-1) c_{0}^{2} / r^{2}, \\
& \mathcal{U}_{3}=n(n-1)(n-2) c_{0}^{3} / r^{3},
\end{aligned}
$$




$$
\begin{aligned}
\mathcal{U}_{4}= & n(n-1)(n-2)(n-3) c_{0}^{4} / r^{4}, \\
f(r)= & k+\frac{16 \pi P}{(n+1) n} r^{2}-\frac{16 \pi M}{n \omega_{n} r^{n-1}}+\frac{(16 \pi Q)^{2}}{2 n(n-1) \omega_{n}^{2} r^{2(n-1)}}+\frac{c_{0} c_{1} m^{2}}{n} r+c_{0}^{2} c_{2} m^{2} \\
& +\frac{(n-1) c_{0}^{3} c_{3} m^{2}}{r}+\frac{(n-1)(n-2) c_{0}^{4} c_{4} m^{2}}{r^{2}},
\end{aligned}
$$

where $M$ and $Q$ are the mass and the charge of the black hole respectively while $\omega_{n}$ denotes the volume of the space spanned by coordinates $x^{i}$ (note that here we use the notation $\omega_{n}$ instead of $V_{n}$ in ref. [29] to avoid confusion with the notation of thermodynamic volume in this paper). In the extended phase space, the pressure $P$ has been identified as $\frac{n(n+1)}{16 \pi l^{2}}$ and the thermodynamic volume is defined as its conjugate quantity. Solving the equation $f\left(r_{\mathrm{h}}\right)=0$ for the largest root, one can express the mass into the function of the horizon radius $r_{\mathrm{h}}$ as $[29,32]$

$$
\begin{aligned}
M= & \frac{n \omega_{n} r_{\mathrm{h}}^{n-1}}{16 \pi}\left[k+\frac{16 \pi P}{(n+1) n} r_{\mathrm{h}}^{2}+\frac{(16 \pi Q)^{2}}{2 n(n-1) \omega_{n}^{2} r_{\mathrm{h}}^{2(n-1)}}+\frac{c_{0} c_{1} m^{2}}{n} r_{\mathrm{h}}+c_{0}^{2} c_{2} m^{2}\right. \\
& \left.+\frac{(n-1) c_{0}^{3} c_{3} m^{2}}{r_{\mathrm{h}}}+\frac{(n-1)(n-2) c_{0}^{4} c_{4} m^{2}}{r_{\mathrm{h}}^{2}}\right] .
\end{aligned}
$$

The Hawking temperature $T$, the entropy $S$, the thermodynamic volume $V$, and the electric potential $\Phi$ have been derived as $[29,32]$

$$
\begin{aligned}
T= & \frac{1}{4 \pi} f^{\prime}\left(r_{\mathrm{h}}\right)=\frac{1}{4 \pi r_{\mathrm{h}}}\left[(n-1) k+\frac{16 \pi P}{n} r_{\mathrm{h}}^{2}-\frac{(16 \pi Q)^{2}}{2 n \omega_{n}^{2} r_{\mathrm{h}}^{2(n-1)}}+c_{0} c_{1} m^{2} r_{\mathrm{h}}+(n-1) c_{0}^{2} c_{2} m^{2}\right. \\
& \left.+\frac{(n-1)(n-2) c_{0}^{3} c_{3} m^{2}}{r_{\mathrm{h}}}+\frac{(n-1)(n-2)(n-3) c_{0}^{4} c_{4} m^{2}}{r_{\mathrm{h}}^{2}}\right] \\
S= & \int_{0}^{r_{\mathrm{h}}} T^{-1}\left(\frac{\partial H}{\partial r}\right)_{Q, P} d r=\frac{\omega_{n}}{4} r_{\mathrm{h}}^{n} \\
V= & \left(\frac{\partial H}{\partial P}\right)_{S, Q}=\frac{\omega_{n}}{n+1} r_{\mathrm{h}}^{n+1}, \\
\Phi= & \left(\frac{\partial H}{\partial Q}\right)_{S, P}=\frac{16 \pi}{(n-1) \omega_{n} r_{\mathrm{h}}^{n-1}} Q .
\end{aligned}
$$

The first law and the Smarr relation of the black hole have been obtained in the extended phase space as [32]

$$
\begin{aligned}
\mathrm{d} H= & T \mathrm{~d} S+V \mathrm{~d} P+\Phi \mathrm{d} Q+\frac{\omega_{n} c_{0} m^{2} r_{\mathrm{h}}^{n}}{16 \pi} \mathrm{d} c_{1}+\frac{n \omega_{n} c_{0}^{2} m^{2} r_{\mathrm{h}}^{n-1}}{16 \pi} \mathrm{d} c_{2} \\
& +\frac{n(n-1) \omega_{n} c_{0}^{3} m^{2} r_{\mathrm{h}}^{n-2}}{16 \pi} \mathrm{d} c_{3}+\frac{n(n-1)(n-2) \omega_{n} c_{0}^{4} m^{2} r_{\mathrm{h}}^{n-3}}{16 \pi} \mathrm{d} c_{4} \\
(n-1) H= & n T S-2 P V+(n-1) \Phi Q-\frac{\omega_{n} c_{0} c_{1} m^{2}}{16 \pi} r_{\mathrm{h}}^{n} \\
& +\frac{n(n-1) \omega_{n} c_{0}^{3} c_{3} m^{2}}{16 \pi} r_{\mathrm{h}}^{n-2}+\frac{n(n-1)(n-2) \omega_{n} c_{0}^{4} c_{4} m^{2}}{8 \pi} r_{\mathrm{h}}^{n-3}
\end{aligned}
$$




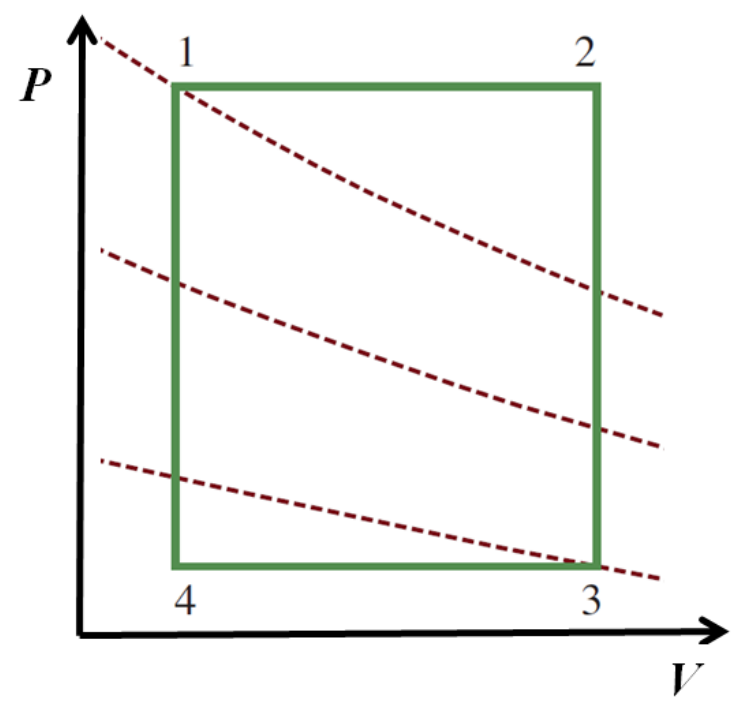

(a)

Figure 1. The heat engine cycle considered.

\section{Four-dimensional charged black holes in massive gravity}

For the four-dimensional black hole (corresponding to $n=2), \mathcal{U}_{3}=\mathcal{U}_{4}=0$ and $c_{3}, c_{4}$ can be set to zero for convenience. Then eq. (2.7) reduces to

$$
M=\frac{\omega_{2} r_{\mathrm{h}}}{8 \pi}\left[k+\frac{8 \pi P}{3} r_{\mathrm{h}}^{2}+\frac{(8 \pi Q)^{2}}{\omega_{2}^{2} r_{\mathrm{h}}^{2}}+\frac{c_{0} c_{1} m^{2}}{2} r_{\mathrm{h}}+c_{0}^{2} c_{2} m^{2}\right] .
$$

Now we define a new kind of heat engine via four-dimensional black holes in massive gravity. As in former literatures [5-7], a rectangle cycle in the $P-V$ plane which consists of two isobars and two isochores is considered here. A sketch picture of the cycle is shown in figure 1 . Note that the subscripts 1,2,3,4 denote the relevant physical quantities evaluated at the four corners 1,2,3,4 respectively.

It can be witnessed from eqs. (2.9) and (2.10) that the entropy $S$ and the thermodynamic volume $V$ are not independent. It is not difficult to conclude that the specific heat at constant volume $C_{V}$ equals to zero. Then the isochores are adiabatic.

Via eqs. (2.8) and (2.9), $T$ can be expressed into the function of $S$ as

$$
T=\frac{-16 \pi^{2} Q^{2}+32 P \pi S^{2}+k S \omega_{2}+c_{0} m^{2}\left(c_{0} c_{2} S \omega_{2}+2 c_{1} S \sqrt{S \omega_{2}}\right)}{8 \pi S \sqrt{S \omega_{2}}} .
$$

Then the specific heat at constant pressure $C_{P}$ can be derived as

$$
C_{P}=T\left(\frac{\partial S}{\partial T}\right)_{P}=\frac{2 S\left[-16 \pi^{2} Q^{2}+32 P \pi S^{2}+k S \omega_{2}+c_{0} m^{2}\left(c_{0} c_{2} S \omega_{2}+2 c_{1} S \sqrt{S \omega_{2}}\right)\right]}{48 \pi^{2} Q^{2}+32 P \pi S^{2}-\left(k+c_{0}^{2} c_{2} m^{2}\right) S \omega_{2}} .
$$

And the heat input $Q_{H}$ can be derived through

$$
Q_{H}=\int_{T_{1}}^{T_{2}} C_{P} d T=\int_{S_{1}}^{S_{2}} C_{P}\left(\frac{\partial T}{\partial S}\right)_{P} d S=\int_{S_{1}}^{S_{2}} T d S=\int_{H_{1}}^{H_{2}} d H=M_{2}-M_{1},
$$


where we have adopted the conjecture that the mass $M$ should be interpreted as the enthalpy $H$. The output heat $Q_{c}$ can be derived in a similar way. Some comments are made in order. The high temperature expansion technique is not necessary here. Both the method utilizing the integral and the method utilizing the exact formula $\eta=1-\frac{M_{3}-M_{4}}{M_{2}-M_{1}}$ proposed in [15] can lead to exact result. Both methods are equivalent to each other. However, when one deal with the scheme where $\left(T_{1}, T_{2}, P_{1}, P_{4}\right)$ or $\left(T_{2}, T_{4}, V_{2}, V_{4}\right)$ are specified as operating parameters in the heat engine cycle, only numerical (not analytical) results can be obtained from these two methods. Because in most cases it is also not easy to express the mass $M$ into the function of $T$. One has to solve the equation for numerical results of $S$ or $r_{\mathrm{h}}$ and then substituting them into the expression of $M$.

Note that the discussion in this paper is not carried out in the ensemble with fixed mass because according to the exact formula $\eta=1-\frac{M_{3}-M_{4}}{M_{2}-M_{1}}$, the heat engine efficiency is always the same as long as $M_{1}, \ldots M_{4}$ are fixed in such an ensemble, making the probe in different backgrounds meaningless. Instead, we follow the scheme where $\left(P_{1}, P_{4}, S_{1}, S_{2}\right)$ are specified as operating parameters in the heat engine cycle. In fact, this treatment is equivalent to the scheme utilized in refs. $[18,20]$ where $\left(P_{1}, P_{4}, V_{1}, V_{2}\right)$ are specified as operating parameters according to eqs. (2.9) and (2.10).

Utilizing eqs. (3.2) and (3.4) (or utilizing eqs. (2.9), (3.1) and (3.4) equivalently), the result of $Q_{H}$ can be calculated as

$$
\begin{aligned}
Q_{H}= & \frac{1}{12 \pi \omega_{2}}\left[3 c_{0} c_{1} m^{2} \omega_{2}\left(S_{2}-S_{1}\right)+3\left(k+c_{0}^{2} c_{2} m^{2}\right) \omega_{2}^{3 / 2}\left(\sqrt{S_{2}}-\sqrt{S_{1}}\right)\right. \\
& \left.+48 \pi^{2} Q^{2} \sqrt{\omega_{2}}\left(S_{2}^{-1 / 2}-S_{1}^{-1 / 2}\right)+32 P_{1} \pi \sqrt{\omega_{2}}\left(S_{2}^{3 / 2}-S_{1}^{3 / 2}\right)\right] .
\end{aligned}
$$

The work done along the cycle can be derived as

$$
W=\left(V_{2}-V_{1}\right)\left(P_{1}-P_{4}\right)=\frac{8}{3 \sqrt{\omega_{2}}}\left(P_{1}-P_{4}\right)\left(S_{2}^{3 / 2}-S_{1}^{3 / 2}\right) .
$$

Then the heat engine efficiency for four-dimensional charged black holes in massive gravity can be calculated as

$$
\eta=\frac{W}{Q_{H}}=\left(1-\frac{P_{4}}{P_{1}}\right) \times \frac{32 P_{1} \pi\left(S_{1}+S_{2}+\sqrt{S_{1} S_{2}}\right)}{B\left(S_{1}, S_{2}, P_{1}\right)}
$$

where

$B\left(S_{1}, S_{2}, P_{1}\right)=32 P_{1} \pi\left(S_{1}+S_{2}+\sqrt{S_{1} S_{2}}\right)-\frac{48 \pi^{2} Q^{2}}{\sqrt{S_{1} S_{2}}}+3 k \omega_{2}+3 c_{0} m^{2} \sqrt{\omega_{2}}\left[c_{0} c_{2} \sqrt{\omega_{2}}+c_{1}\left(\sqrt{S_{2}}+\sqrt{S_{1}}\right)\right]$.

It can be examined that this result agrees very well with the result obtained via the exact efficiency formula $\eta=1-\frac{M_{3}-M_{4}}{M_{2}-M_{1}}$.

From eq. (3.8), it can be witnessed clearly that the effect of massive gravity is reflected in the fourth term. When $c_{1}<0, c_{2}<0$, the heat engine have a higher efficiency for the cases $m^{2}>0$ than for the case $m=0$. In other words, the graviton mass might help improve the heat engine efficiency. 


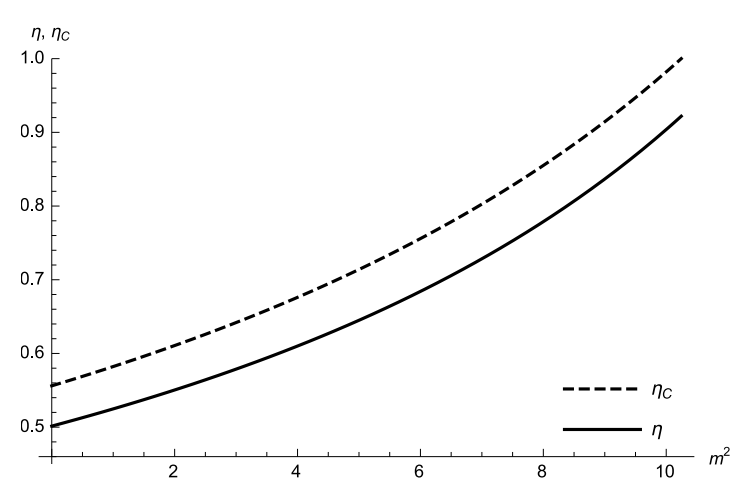

(a)

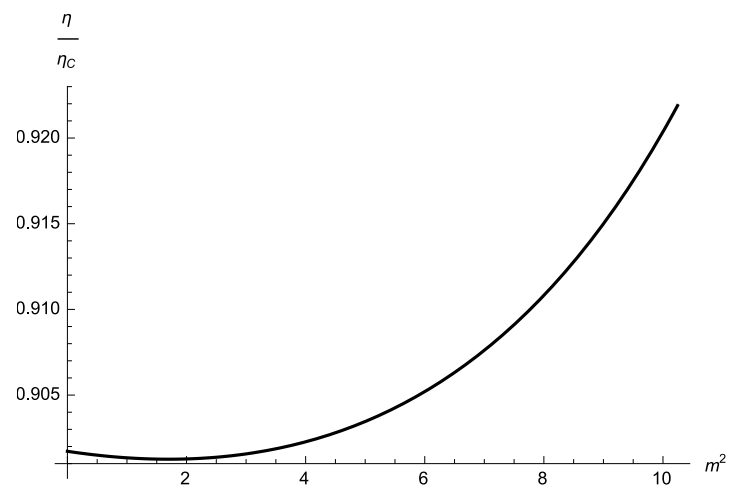

(b)

Figure 2. A specific example of four-dimensional charged black holes with the parameters chosen as $k=1, c_{0}=1, c_{1}=-2, c_{2}=-3, P_{1}=2, P_{4}=1, Q=1, S_{1}=8, S_{2}=10$. (a) $\eta, \eta_{C}$ as the function of $m^{2}$. (b) $\eta / \eta_{C}$ as the function of $m^{2}$.

To sketch a picture of this issue, one can consider a specific example with the parameters chosen as $k=1, c_{0}=1, c_{1}=-2, c_{2}=-3, P_{1}=2, P_{4}=1, S_{1}=8, S_{2}=10$. To observe the effect of $m^{2}$ on the heat engine efficiency, we can fix the charge $Q=1$ and let $m^{2}$ vary from 0 to $m_{0}^{2}\left(m_{0}^{2}\right.$ is the maximum value of $m^{2}$ that can ensure the positivity of the Hawking temperature). The efficiency $\eta$ as the function of $m^{2}$ is plotted in figure $2 \mathrm{a}$. It can be witnessed clearly that the efficiency increases with $m^{2}$. Here, $m_{0}^{2}$ can be determined from eq. (3.2) as 10.2445. The corresponding maximum efficiency can be obtained as 0.9219 while the efficiency for $m=0$ takes the value 0.5014 . The existence of graviton mass has improved the heat engine efficiency significantly.

On the other hand, we can fix $m^{2}=1$ and let $Q$ vary from 0 to $Q_{0}\left(Q_{0}^{2}\right.$ is the maximum value of $Q$ that can ensure the positivity of the Hawking temperature) to check the effect of charge when $m^{2}>0$. From figure 3a, one can see clearly that the efficiency also increases with $Q . Q_{0}^{2}$ can be determined from eq. (3.2) as 6.1187. The corresponding maximum efficiency can be obtained as 0.8381 while the efficiency for $Q=0$ takes the value 0.5194 .

To end the discussions here, we would like to remind the reader that one may possibly make a mistake to obtain an efficiency higher than 1 (obviously not physical) if he does not consider the constraint on the $m^{2}$ and $Q$ set by the positivity of the Hawking temperature.

It is also of interest to compare the heat engine efficiency with the well-known Carnot efficiency $\eta_{C}$, whose formula reads

$$
\eta_{C}=1-\frac{T_{C}}{T_{H}}
$$

where $T_{C}, T_{H}$ denote the lowest and the highest temperature of the heat engine cycle. For the cycle considered in this paper, $T_{H}=T_{2}$ and $T_{C}=T_{4}$. They can be obtained by substituting $S=S_{2}, P=P_{1}$ and $S=S_{1}, P=P_{4}$ respectively into the eq. (3.2). From figures $2 \mathrm{a}$ and $3 \mathrm{a}$, it can be observed that $\eta_{C}$ increases with $m^{2}$ and $Q$. When $Q=1$, the maximum $\eta_{C}$ can approach 1 while the value for $m^{2}=0$ reads 0.5561 . When $m^{2}=1$, the maximum $\eta_{C}$ also approaches 1 while the value for $Q=0$ reads 0.5742 . The behavior of 


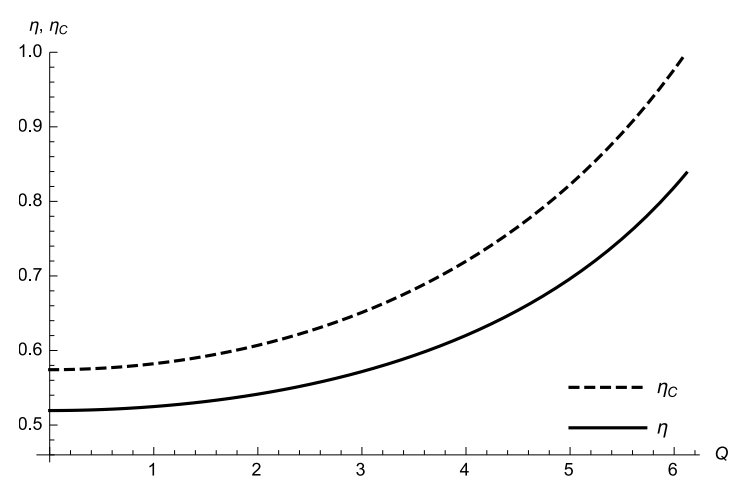

(a)

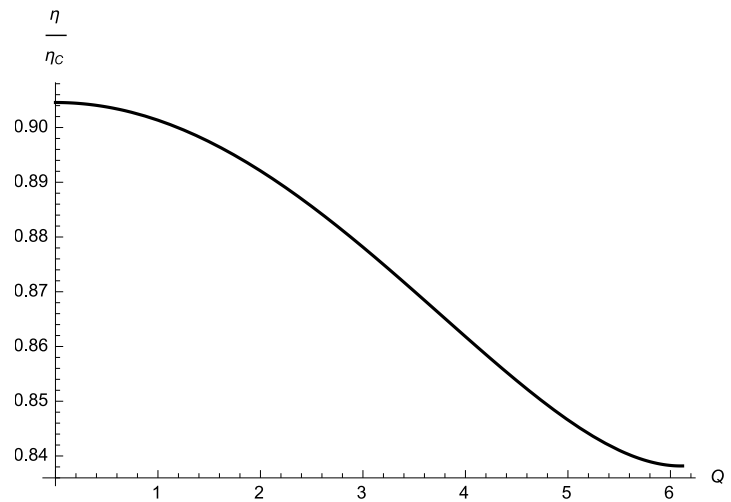

(b)

Figure 3. A specific example of four-dimensional charged black holes with the parameters chosen as $k=1, c_{0}=1, c_{1}=-2, c_{2}=-3, P_{1}=2, P_{4}=1, m^{2}=1, S_{1}=8, S_{2}=10$. (a) $\eta, \eta_{C}$ as the function of $Q$. (b) $\eta / \eta_{C}$ as the function of $Q$.

$\eta$ approaching $\eta_{C}$ is depicted in figures $2 \mathrm{~b}$ and $3 \mathrm{~b}$. With the increasing of $m^{2}$, the ratio $\eta / \eta_{C}$ first decreases to a minimum value and then increases monotonically to approach the maximum value 0.9219 . However, the effect of $Q$ is quite the reverse. The ratio decreases with $Q$.

\section{Five-dimensional neutral black holes in massive gravity}

For the five-dimensional black hole (corresponding to $n=3$ ), $\mathcal{U}_{4}=0$ and $c_{4}$ can be set to zero for convenience. Unlike the four-dimensional case discussed in the former section, $c_{3} \neq 0$. For simplicity, we consider the neutral black holes here (corresponding to $Q=0$ ). Then eq. (2.7) becomes

$$
M=\frac{3 \omega_{3} r_{\mathrm{h}}^{2}}{16 \pi}\left[k+\frac{4 \pi P}{3} r_{\mathrm{h}}^{2}+\frac{c_{0} c_{1} m^{2}}{3} r_{\mathrm{h}}+c_{0}^{2} c_{2} m^{2}+\frac{2 c_{0}^{3} c_{3} m^{2}}{r_{\mathrm{h}}}\right] .
$$

In this section, we would construct the heat engine model via five-dimensional neutral black holes in massive gravity. And a similar rectangle cycle in the $P-V$ plane will be considered.

By the same token, one can quickly get $C_{V}=0$, implying that no heat flows along the isochores.

Utilizing eqs. (2.8) and (2.9), the Hawking temperature of five-dimensional neutral black holes can be obtained as

$$
T=\frac{32 \times 2^{1 / 3} P \pi S+6 k\left(S \omega_{3}^{2}\right)^{1 / 3}+3 c_{0} m^{2}\left[2^{1 / 3} c_{0}^{2} c_{3} \omega_{3}+2^{2 / 3} c_{1}\left(S^{2} \omega_{3}\right)^{1 / 3}+2 c_{0} c_{2}\left(S \omega_{3}^{2}\right)^{1 / 3}\right]}{12 \times 2^{2 / 3} \pi\left(S^{2} \omega_{3}\right)^{1 / 3}} .
$$

The specific heat at constant pressure $C_{P}$ can be obtained via $C_{P}=T\left(\frac{\partial S}{\partial T}\right)_{P}$ as

$C_{P}=\frac{3 S\left(S \omega_{3}^{2}\right)^{2 / 3}\left\{32 \times 2^{1 / 3} P \pi S+6 k\left(S \omega_{3}^{2}\right)^{1 / 3}+3 c_{0} m^{2}\left[2^{1 / 3} c_{0}^{2} c_{3} \omega_{3}+2^{2 / 3} c_{1}\left(S^{2} \omega_{3}\right)^{1 / 3}+2 c_{0} c_{2}\left(S \omega_{3}^{2}\right)^{1 / 3}\right]\right\}}{32 \times 2^{1 / 3} P \pi S\left(S \omega_{3}^{2}\right)^{2 / 3}-6 k S \omega_{3}^{2}-6 c_{0}^{2} c_{2} m^{2} S \omega_{3}^{2}-6 \times 2^{1 / 3} c_{0}^{3} c_{3} m^{2} \omega_{3}\left(S \omega_{3}^{2}\right)^{2 / 3}}$. 
Utilizing eqs. (3.4) and (4.2) (or utilizing eqs. (2.9), (3.4) and (4.1) equivalently), the result of $Q_{H}$ can be calculated as

$$
\begin{aligned}
Q_{H}= & M_{2}-M_{1}=\frac{1}{8 \pi}\left[8 \times 2^{2 / 3} P_{1} \pi \omega_{3}^{-1 / 3}\left(S_{2}^{4 / 3}-S_{1}^{4 / 3}\right)+3 \times 2^{1 / 3} k \omega_{3}^{1 / 3}\left(S_{2}^{2 / 3}-S_{1}^{2 / 3}\right)+2 c_{0} c_{1} m^{2}\left(S_{2}-S_{1}\right)\right. \\
& \left.+3 \times 2^{2 / 3} c_{0}^{3} c_{3} m^{2} \omega_{3}^{2 / 3}\left(S_{2}^{1 / 3}-S_{1}^{1 / 3}\right)+3 \times 2^{1 / 3} c_{0}^{2} c_{2} m^{2} \omega_{3}^{1 / 3}\left(S_{2}^{2 / 3}-S_{1}^{2 / 3}\right)\right] .
\end{aligned}
$$

The work $W$ can be obtained as

$$
W=\left(V_{2}-V_{1}\right)\left(P_{1}-P_{4}\right)=2^{2 / 3} \omega_{3}^{-1 / 3}\left(P_{1}-P_{4}\right)\left(S_{2}^{4 / 3}-S_{1}^{4 / 3}\right) .
$$

And the heat engine efficiency $\eta$ for five-dimensional neutral black holes can be derived as

$$
\eta=\frac{W}{Q_{H}}=\left(1-\frac{P_{4}}{P_{1}}\right) \times \frac{8 \times 2^{2 / 3} P_{1} \pi\left(S_{2}^{4 / 3}-S_{1}^{4 / 3}\right)}{C\left(S_{1}, S_{2}, P_{1}\right)},
$$

where

$$
\begin{aligned}
C\left(S_{1}, S_{2}, P_{1}\right)= & c_{0} \omega_{3}^{1 / 3} m^{2}\left[2 c_{1}\left(S_{2}-S_{1}\right)+3 \times 2^{2 / 3} c_{0}^{2} c_{3} \omega_{3}^{2 / 3}\left(S_{2}^{1 / 3}-S_{1}^{1 / 3}\right)+3 \times 2^{1 / 3} c_{0} c_{2} \omega_{3}^{1 / 3}\left(S_{2}^{2 / 3}-S_{1}^{2 / 3}\right)\right] \\
& +8 \times 2^{2 / 3} P_{1} \pi\left(S_{2}^{4 / 3}-S_{1}^{4 / 3}\right)+3 \times 2^{1 / 3} k \omega_{3}^{2 / 3}\left(S_{2}^{2 / 3}-S_{1}^{2 / 3}\right) .
\end{aligned}
$$

From eq. (4.7), it can be witnessed clearly that the effect of massive gravity is reflected in the first term which contains the factor $\mathrm{m}^{2}$. Obviously, this case is more complicated than the four-dimensional case. Not only the $c_{1}, c_{2}, m^{2}$ exert influence on the efficiency, but also the constant $c_{3}$ corresponding to the third massive potential contributes to the efficiency. When $c_{1}<0, c_{2}<0, c_{3}<0$, the heat engine efficiency of the cases $m^{2}>0$ are higher than that of the case $m=0$, just as in the four-dimensional charged black holes discussed in the former section.

To gain an intuitive understanding, we present a specific example here with the parameters chosen as $k=1, c_{0}=1, c_{1}=-2, c_{2}=-3, c_{3}=-4, P_{1}=2, P_{4}=1, S_{1}=8, S_{2}=10$. To guarantee the positivity of the Hawking temperature, one can first determine the maximum value of $m^{2}$ (1.6571) via eq. (4.2). Then one can let $m^{2}$ vary from 0 to this maximum value to probe how the efficiency is affected by different choices of $m^{2}$. The efficiency graph is plotted in figure $4 \mathrm{a}$, from which one can observe that the efficiency increases with $\mathrm{m}^{2}$. The maximum efficiency can be obtained as 0.9217 comparing to 0.4808 for $m=0$, showing the impact of the graviton mass on the heat engine efficiency.

One can also make some comparison with Carnot efficiency $\eta_{C}$, whose behavior is also plotted in figure 4a. As in the four-dimensional charged case, $\eta_{C}$ increases with $m^{2}$. The ratio $\eta / \eta_{C}$ first decreases monotonically and then increases, which can be witnessed clearly from figure $4 \mathrm{~b}$. However, the maximum value $(0.9356)$ of the ratio $\eta / \eta_{C}$ is obtained when $m^{2}=0$, different from four-dimensional charged case.

Note that there exists a minimum value of $\eta / \eta_{C}$ in both figures $2 \mathrm{~b}$ and $4 \mathrm{~b}$. These minimum values set lower bounds for the possibility that the heat engine efficiency approaches the Carnot efficiency. And these lower bounds may be related to some deep physics which certainly deserves to be disclosed in the future research. Probing this issue from the context of AdS/CFT may be a promising direction. 


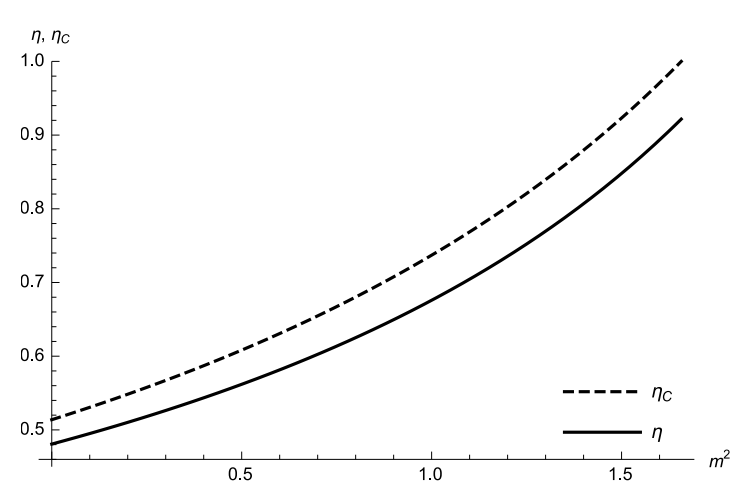

(a)

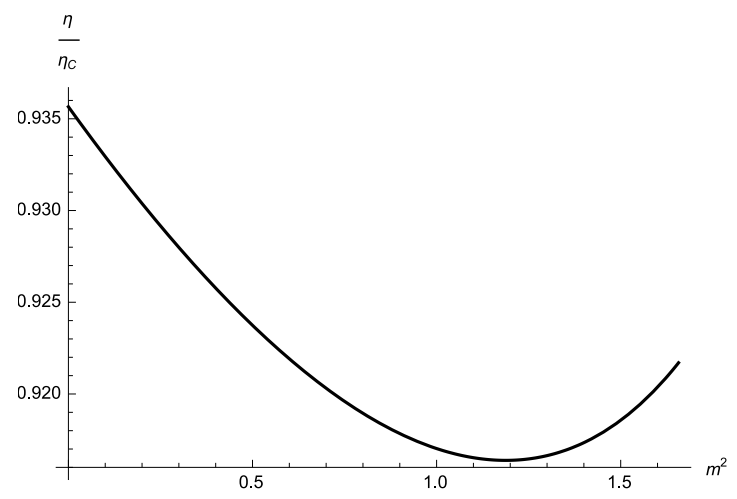

(b)

Figure 4. A specific example of five-dimensional neutral black holes with the parameters chosen as $k=1, c_{0}=1, c_{1}=-2, c_{2}=-3, c_{3}=-4, P_{1}=2, P_{4}=1, S_{1}=8, S_{2}=10$ (a) $\eta, \eta_{C}$ as the function of $m^{2}$ (b) $\eta / \eta_{C}$ as the function of $m^{2}$.

\section{Conclusions}

Heat engine models are constructed within the framework of massive gravity. Specifically, we define new kinds of heat engine via four-dimensional charged black holes and fivedimensional neutral black holes in massive gravity. A rectangle cycle in the $P-V$ plane which consists of two isobars and two isochores is considered. The heat input and the work done along the cycle are calculated and the heat engine efficiency is obtained. We argue that both the method utilizing the integral and the method utilizing the exact formula proposed in [15] can lead to exact result and the high temperature expansion technique is not necessary. Both methods are equivalent to each other. However, when one deal with the scheme where $\left(T_{1}, T_{2}, P_{1}, P_{4}\right)$ or $\left(T_{2}, T_{4}, V_{2}, V_{4}\right)$ are specified as operating parameters in the heat engine cycle, only numerical (not analytical) results can be obtained from these two methods. Because in most cases it is also not easy to express the mass $M$ into the function of $T$. One has to solve the equation for numerical results of $S$ or $r_{\mathrm{h}}$ and then substituting them into the expression of $M$.

The effect of massive gravity on the heat engine efficiency is probed. For the fourdimensional charged black holes in massive gravity, it is shown that the heat engine have a higher efficiency for the cases $m^{2}>0$ than for the case $m=0$ when $c_{1}<0, c_{2}<0$. In other words, the graviton mass can help improve the heat engine efficiency. A specific example with the parameters chosen as $k=1, c_{0}=1, c_{1}=-2, c_{2}=-3, P_{1}=2, P_{4}=$ $1, S_{1}=8, S_{2}=10$ is presented. We fix the charge $Q=1$ and let $m^{2}$ vary from 0 to $m_{0}^{2}\left(m_{0}^{2}\right.$ is the maximum value of $m^{2}$ that can ensure the positivity of the Hawking temperature). It is shown graphically that the efficiency increases with $\mathrm{m}^{2}$. The maximum efficiency can be obtained as 0.9219 while the efficiency for $m=0$ takes the value 0.5014 . The existence of graviton mass has improved the heat engine efficiency significantly.

The case of the five-dimensional neutral black hole is more complicated comparing to the four-dimensional case. Not only the $c_{1}, c_{2}, m^{2}$ exert influence on the efficiency, but also 
the constant $c_{3}$ corresponding to the third massive potential contributes to the efficiency. When $c_{1}<0, c_{2}<0, c_{3}<0$, the heat engine efficiency of the cases $m^{2}>0$ are higher than that of the case $m=0$. We present an example with the parameters chosen as $k=1, c_{0}=1, c_{1}=-2, c_{2}=-3, c_{3}=-4, P_{1}=2, P_{4}=1, S_{1}=8, S_{2}=10$. The maximum efficiency can be obtained as 0.9217 comparing to 0.4808 for $m=0$, showing the impact of the graviton mass on the heat engine efficiency.

It is also of interest to compare the heat engine efficiency with the Carnot efficiency. For the four-dimensional black holes, with the increasing of $m^{2}$, the ratio $\eta / \eta_{C}$ first decreases to a minimum value and then increases monotonically to approach the maximum value 0.9219. However, the effect of $Q$ is quite the reverse. The ratio decreases with $Q$. This peculiar property may be attributed to the combined effect of the charge and the massive gravity. The qualitative behavior of $\eta / \eta_{C}$ is similar for the five-dimensional neutral black hole case. However, the maximum value of the ratio $\eta / \eta_{C}$ is obtained when $m^{2}=0$, different from four-dimensional case.

\section{Acknowledgments}

The authors would like to express their sincere gratitude to both the editor and the referee whose efforts have help improved the quality of this manuscript significantly. We also want to thank Ya-Peng Hu, Shao-Wen Wei, Xing-Hui Feng, Peng Liu and Shan-Quan Lan for valuable discussions. This research is supported by National Natural Science Foundation of China (Grant No. 11605082). The authors are in part supported by Natural Science Foundation of Guangdong Province, China (Grant Nos. 2016A030310363, 2016A030307051, 2015A030313789) and Department of Education of Guangdong Province, China (Grant Nos. 2017KQNCX124,2017KZDXM056).

Open Access. This article is distributed under the terms of the Creative Commons Attribution License (CC-BY 4.0), which permits any use, distribution and reproduction in any medium, provided the original author(s) and source are credited.

\section{References}

[1] B.P. Dolan, Pressure and volume in the first law of black hole thermodynamics, Class. Quant. Grav. 28 (2011) 235017 [arXiv:1106.6260] [INSPIRE].

[2] D. Kubizňák and R.B. Mann, P - V criticality of charged AdS black holes, JHEP 07 (2012) 033 [arXiv: 1205.0559] [INSPIRE].

[3] D. Kastor, S. Ray and J. Traschen, Enthalpy and the Mechanics of AdS Black Holes, Class. Quant. Grav. 26 (2009) 195011 [arXiv:0904.2765] [INSPIRE].

[4] D. Kubizňák, R.B. Mann and M. Teo, Black hole chemistry: thermodynamics with Lambda, Class. Quant. Grav. 34 (2017) 063001 [arXiv:1608.06147] [INSPIRE].

[5] C.V. Johnson, Holographic Heat Engines, Class. Quant. Grav. 31 (2014) 205002 [arXiv: 1404.5982] [INSPIRE].

[6] C.V. Johnson, Gauss-Bonnet black holes and holographic heat engines beyond large N, Class. Quant. Grav. 33 (2016) 215009 [arXiv:1511.08782] [INSPIRE]. 
[7] C.V. Johnson, Born-Infeld AdS black holes as heat engines, Class. Quant. Grav. 33 (2016) 135001 [arXiv: 1512.01746] [INSPIRE].

[8] A. Belhaj, M. Chabab, H. El Moumni, K. Masmar, M.B. Sedra and A. Segui, On Heat Properties of AdS Black Holes in Higher Dimensions, JHEP 05 (2015) 149 [arXiv: 1503.07308] [INSPIRE].

[9] J.-X. Mo, F. Liang and G.-Q. Li, Heat engine in the three-dimensional spacetime, JHEP 03 (2017) 010 [arXiv:1701.00883] [InSPIRE].

[10] R.A. Hennigar, F. McCarthy, A. Ballon and R.B. Mann, Holographic heat engines: general considerations and rotating black holes, Class. Quant. Grav. 34 (2017) 175005 [arXiv: 1704.02314] [INSPIRE].

[11] J. Sadeghi and K. Jafarzade, The Thermodynamic Efficiency in Static and Dynamic Black Holes, Int. J. Theor. Phys. 56 (2017) 3387 [arXiv: 1504.07744] [INSPIRE].

[12] M.R. Setare and H. Adami, Polytropic black hole as a heat engine, Gen. Rel. Grav. 47 (2015) 133 [INSPIRE].

[13] J. Sadeghi and K. Jafarzade, Phase transition and holographic in modified Hor̆ava-Lifshitz black hole, Int. J. Mod. Phys. D 26 (2017) 1750138 [arXiv:1604.02973] [INSPIRE].

[14] C. Bhamidipati and P.K. Yerra, Heat engines for dilatonic Born-Infeld black holes, Eur. Phys. J. C 77 (2017) 534 [arXiv:1606.03223] [InSPIRE].

[15] C.V. Johnson, An Exact Efficiency Formula for Holographic Heat Engines, Entropy 18 (2016) 120 [arXiv:1602.02838] [INSPIRE].

[16] S.-W. Wei and Y.-X. Liu, Implementing black hole as efficient power plant,

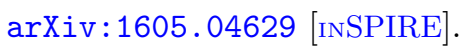

[17] H. Liu and X.-H. Meng, Effects of dark energy on the efficiency of charged AdS black holes as heat engines, Eur. Phys. J. C 77 (2017) 556 [arXiv:1704.04363] [InSPIRE].

[18] C.V. Johnson, Taub-Bolt heat engines, Class. Quant. Grav. 35 (2018) 045001 [arXiv:1705.04855] [INSPIRE].

[19] H. Xu, Y. Sun and L. Zhao, Black hole thermodynamics and heat engines in conformal gravity, Int. J. Mod. Phys. D 26 (2017) 1750151 [arXiv:1706.06442] [INSPIRE].

[20] C.V. Johnson, Approaching the Carnot Limit at Finite Power: An Exact Solution, arXiv:1703.06119 [INSPIRE].

[21] M. Fierz and W. Pauli, On relativistic wave equations for particles of arbitrary spin in an electromagnetic field, Proc. Roy. Soc. Lond. A 173 (1939) 211 [InSPIRE].

[22] D.G. Boulware and S. Deser, Inconsistency of finite range gravitation, Phys. Lett. B 40 (1972) 227 [INSPIRE].

[23] D.G. Boulware and S. Deser, Can gravitation have a finite range?, Phys. Rev. D 6 (1972) 3368 [INSPIRE].

[24] C. de Rham and G. Gabadadze, Generalization of the Fierz-Pauli Action, Phys. Rev. D 82 (2010) 044020 [arXiv: 1007.0443] [InSPIRE].

[25] C. de Rham, G. Gabadadze and A.J. Tolley, Resummation of Massive Gravity, Phys. Rev. Lett. 106 (2011) 231101 [arXiv:1011.1232] [INSPIRE]. 
[26] S.F. Hassan and R.A. Rosen, Resolving the Ghost Problem in non-Linear Massive Gravity, Phys. Rev. Lett. 108 (2012) 041101 [arXiv:1106.3344] [InSPIRE].

[27] S.F. Hassan, R.A. Rosen and A. Schmidt-May, Ghost-free Massive Gravity with a General Reference Metric, JHEP 02 (2012) 026 [arXiv:1109.3230] [INSPIRE].

[28] D. Vegh, Holography without translational symmetry, arXiv:1301.0537 [INSPIRE].

[29] R.-G. Cai, Y.-P. Hu, Q.-Y. Pan and Y.-L. Zhang, Thermodynamics of Black Holes in Massive Gravity, Phys. Rev. D 91 (2015) 024032 [arXiv:1409.2369] [INSPIRE].

[30] A. Adams, D.A. Roberts and O. Saremi, Hawking-Page transition in holographic massive gravity, Phys. Rev. D 91 (2015) 046003 [arXiv:1408.6560] [InSPIRE].

[31] Y.-P. Hu and H. Zhang, Misner-Sharp Mass and the Unified First Law in Massive Gravity, Phys. Rev. D 92 (2015) 024006 [arXiv: 1502.00069] [INSPIRE].

[32] J. Xu, L.-M. Cao and Y.-P. Hu, P - V criticality in the extended phase space of black holes in massive gravity, Phys. Rev. D 91 (2015) 124033 [arXiv:1506.03578] [INSPIRE].

[33] S.H. Hendi, B. Eslam Panah, S. Panahiyan and M. Momennia, Magnetic brane solutions in Gauss-Bonnet-Maxwell massive gravity, Phys. Lett. B 772 (2017) 43 [INSPIRE].

[34] S. Upadhyay, B. Pourhassan and H. Farahani, $P-V$ criticality of first-order entropy corrected AdS black holes in massive gravity, Phys. Rev. D 95 (2017) 106014 [arXiv: 1704.01016] [INSPIRE].

[35] Y.-P. Hu, F. Pan and X.-M. Wu, The effects of massive graviton on the equilibrium between the black hole and radiation gas in an isolated box, Phys. Lett. B 772 (2017) 553 [arXiv: 1703.08599] [INSPIRE].

[36] Y.-P. Hu, X.-M. Wu and H. Zhang, Generalized Vaidya Solutions and Misner-Sharp mass for n-dimensional massive gravity, Phys. Rev. D 95 (2017) 084002 [arXiv:1611.09042] [INSPIRE].

[37] Y.-P. Hu, X.-X. Zeng and H.-Q. Zhang, Holographic Thermalization and Generalized Vaidya-AdS Solutions in Massive Gravity, Phys. Lett. B 765 (2017) 120 [arXiv:1611.00677] [INSPIRE].

[38] X.-X. Zeng, H. Zhang and L.-F. Li, Phase transition of holographic entanglement entropy in massive gravity, Phys. Lett. B 756 (2016) 170 [arXiv:1511.00383] [INSPIRE].

[39] D.-C. Zou, Y. Liu and R.-H. Yue, Behavior of quasinormal modes and Van der Waals-like phase transition of charged AdS black holes in massive gravity, Eur. Phys. J. C 77 (2017) 365 [arXiv: 1702.08118] [INSPIRE].

[40] D.-C. Zou, R.-H. Yue and M. Zhang, Reentrant phase transitions of higher-dimensional AdS black holes in dRGT massive gravity, Eur. Phys. J. C 77 (2017) 256 [arXiv:1612.08056] [INSPIRE].

[41] W.-J. Pan and Y.-C. Huang, Holographic complexity and action growth in massive gravities, Phys. Rev. D 95 (2017) 126013 [arXiv:1612.03627] [INSPIRE].

[42] S.H. Hendi, R.B. Mann, S. Panahiyan and B. Eslam Panah, Van der Waals like behavior of topological AdS black holes in massive gravity, Phys. Rev. D 95 (2017) 021501 [arXiv: 1702.00432] [INSPIRE]. 